\title{
Effect of interpregnancy weight change on perinatal outcomes: systematic review and meta-analysis
}

\author{
Noor E. W. D. Teulings ${ }^{1,2^{*}}$ (D), Katya L. Masconi ${ }^{1}$, Susan E. Ozanne ${ }^{2}$, Catherine E. Aiken ${ }^{2,3}$ and Angela M. Wood
}

\begin{abstract}
Background: Although obesity is a well-known risk factor for adverse pregnancy outcomes, evidence is sparse about the effects of interpregnancy weight change on the risk of adverse perinatal complications in a subsequent pregnancy. The current study aims to assess the effect of interpregnancy weight change on the risk of developing gestational diabetes, pre-eclampsia, pregnancy induced hypertension, preterm birth, or delivering a large- or smallfor-gestational age neonate.

Methods: Pubmed, Ovid Embase, ClinicalTrial.gov and the Cochrane library were systematically searched up until July 24th, 2019. Interpregnancy weight change was defined as the difference between pre-pregnancy weight of an index pregnancy and a consecutive pregnancy. Inclusion criteria included full text original articles reporting quantitative data about interpregnancy weight change in multiparous women with any time interval between consecutive births and the risk of any perinatal complication of interest. Studies reporting adjusted odds ratios and a reference group of -1 to $+1 \mathrm{BMl}$ unit change between pregnancies were harmonised by meta-analysis.
\end{abstract}

Results: Twenty-three cohort studies identified a total of 671,906 women with two or more consecutive pregnancies. Seven of these studies were included in the meta-analysis (280,672 women). Interpregnancy weight gain was consistently associated with a higher risk of gestational diabetes, pre-eclampsia, pregnancy induced hypertension and largefor-gestational age births. In contrast, interpregnancy weight loss was associated with a lower risk of delivering a large-for-gestational age neonate. The effect magnitude (relative risk) of interpregnancy weight gain on pregnancy induced hypertension or delivering a large-for-gestational age neonate was greater among women with a normal BMI in the index pregnancy compared to women with a starting BMl $\geq 25 \mathrm{~kg} / \mathrm{m}^{2}$.

Conclusion: These findings confirm that interpregnancy weight change impacts the risk of developing perinatal complications in a subsequent pregnancy. This provides evidence in support of guidelines encouraging women to achieve post-partum weight loss, as their risk of perinatal complications might be minimised if they return to their pre-pregnancy weight before conceiving again. Prospectively registered with PROSPERO (CRD42017067326).

Keywords: BMI, Hypertensive disorders of pregnancy, Interpregnancy weight change, Meta-analysis, Obesity, Perinatal complications, Systematic review

\footnotetext{
* Correspondence: nt358@medschl.cam.ac.uk

'Department of Public Health and Primary Care, University of Cambridge, 2

Worth's Causeway Cambridge, Cambridge CB1 8RN, UK

${ }^{2}$ University of Cambridge Metabolic Research Laboratories and MRC

Metabolic Diseases Unit, Wellcome Trust-MRC Institute of Metabolic Science,

Addenbrooke's Hospital, Cambridge CB2 0QQ, UK

Full list of author information is available at the end of the article
}

(c) The Author(s). 2019 Open Access This article is distributed under the terms of the Creative Commons Attribution 4.0 International License (http://creativecommons.org/licenses/by/4.0/), which permits unrestricted use, distribution, and reproduction in any medium, provided you give appropriate credit to the original author(s) and the source, provide a link to the Creative Commons license, and indicate if changes were made. The Creative Commons Public Domain Dedication waiver (http://creativecommons.org/publicdomain/zero/1.0/) applies to the data made available in this article, unless otherwise stated. 


\section{Background}

Obesity is an increasing global health concern, with more than 1.9 billion adults worldwide being overweight [1] and approximately one in two US women of childbearing age now being considered overweight or obese [2]. Considerable evidence exists showing serious perinatal complications associated with obesity in pregnancy including gestational diabetes (GDM), pre-eclampsia (PE) and neonatal death [3]. There is also an increased risk of complications such as fetal growth restriction and preterm birth amongst underweight women [4]. However, evidence is sparse about the effect of interpregnancy weight change on the risk of adverse outcomes in subsequent pregnancies. Current NICE guidelines in the UK recommend that overweight or obese women are referred for weight loss support at the 6-8 week postnatal check-up [5] despite limited evidence to support widespread implementation of such health promotion strategies and of benefit for future pregnancy outcomes [6].

The current study aimed to systematically synthesise the published evidence on the associations between interpregnancy weight change and common perinatal complications for both mother and child including GDM, PE, pregnancy induced hypertension (PIH), preterm birth (PTB), and delivery of a large- and small-for-gestational age neonate (LGA and SGA). Additionally, we compared the risk of these complications after interpregnancy weight change in women with a normal BMI and overweight or obese women, and where possible, we investigated the dose-response relationships.

\section{Methods}

Eligibility criteria, information sources, search strategy

The electronic databases PubMed, Ovid EMBASE, ClinicalTrials.gov and Cochrane Central were systematically searched until July 24th, 2019. The search strategy included terms relating to 'interpregnancy', 'between pregnancy', 'weight change' or 'BMI'. These search terms were combined with the outcomes of interest ('gestational diabetes', 'pre-eclampsia', 'pregnancy-induced hypertension', 'preterm birth', 'small-for-gestational age' and 'largefor-gestational age') and synonyms of these outcomes (for full search string see Additional file 3: Table S1). Furthermore, we cross-referenced selected papers for additional articles to include. The studies identified were uploaded onto Covidence, an online tool for screening of papers for

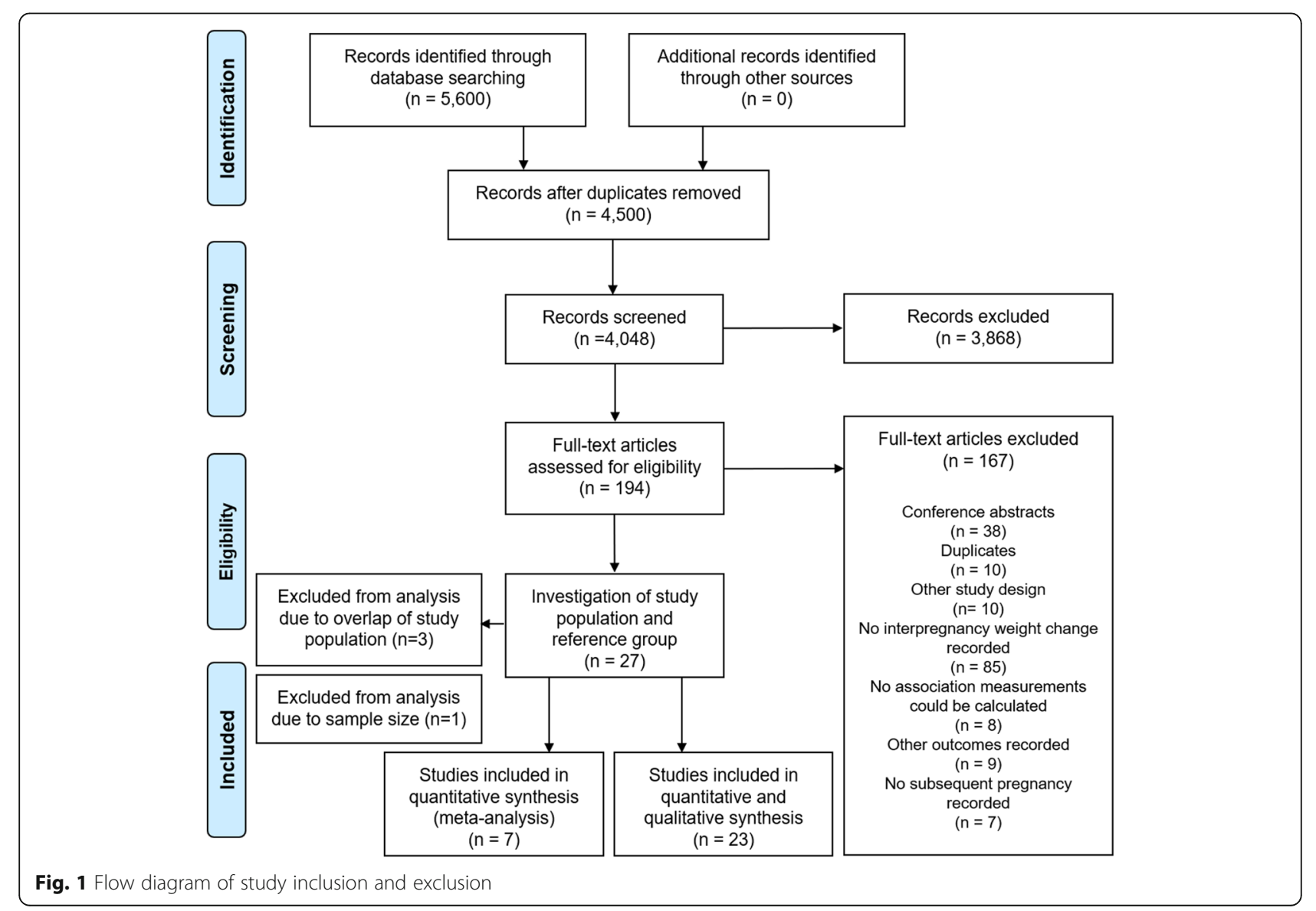




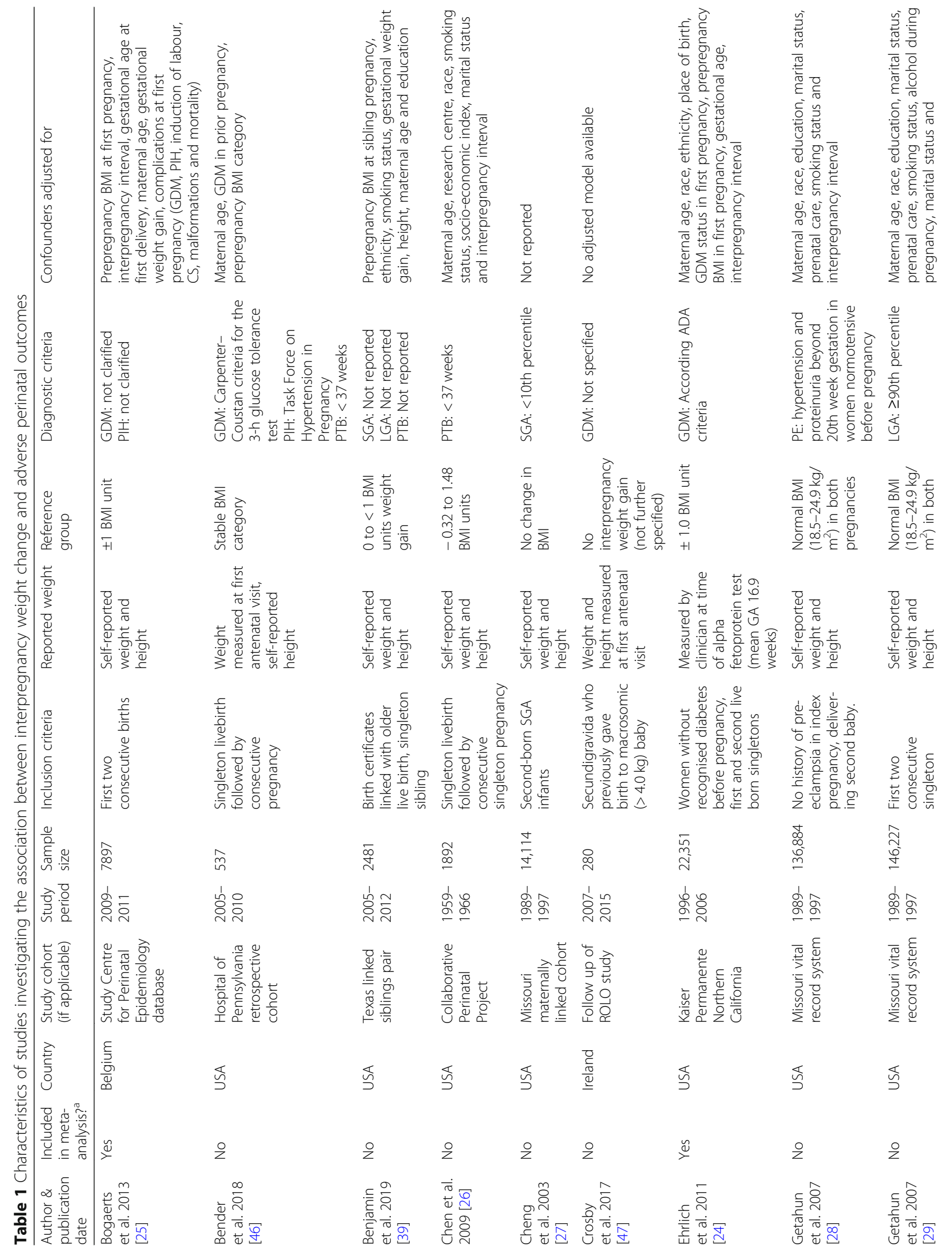




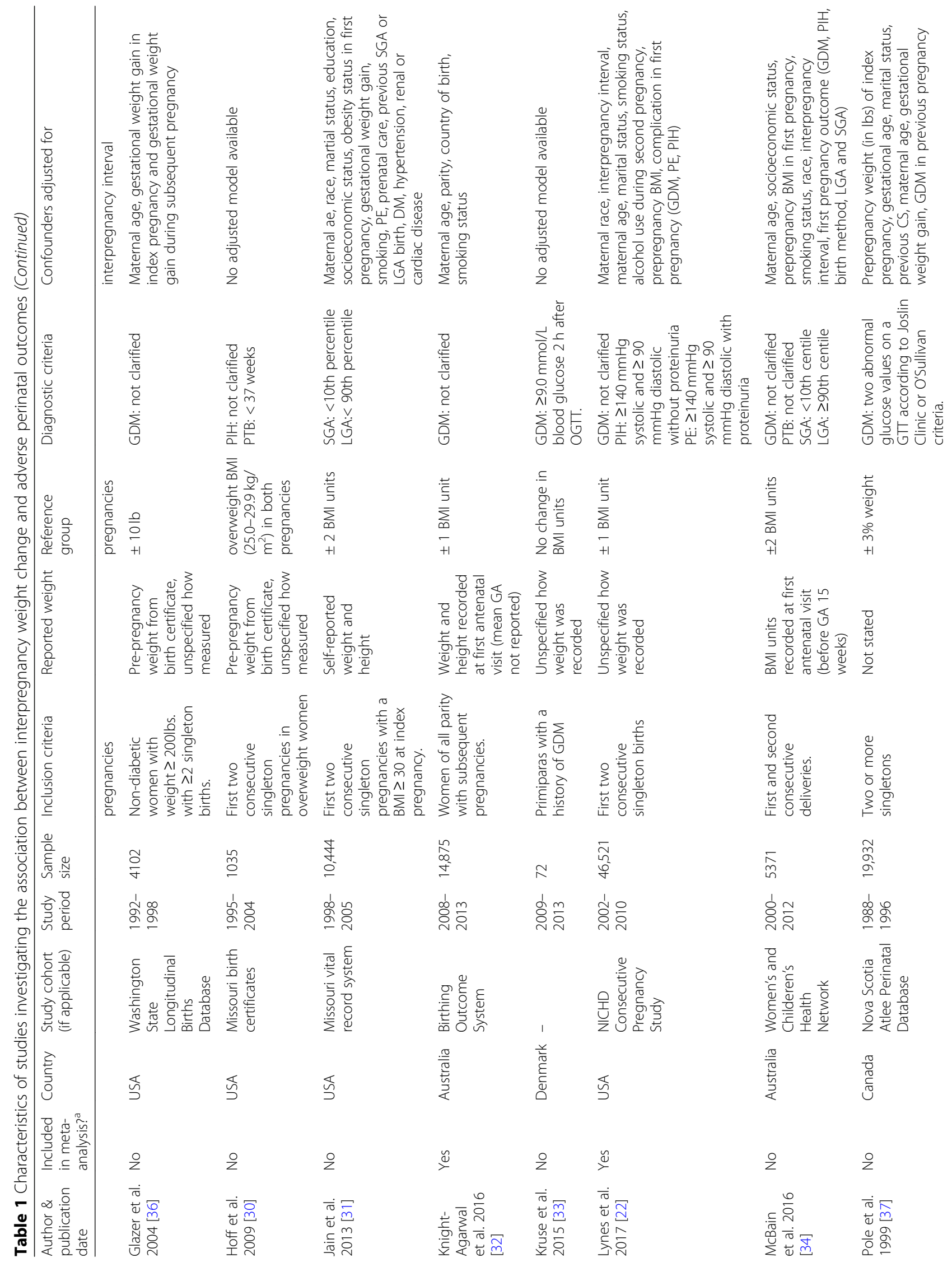




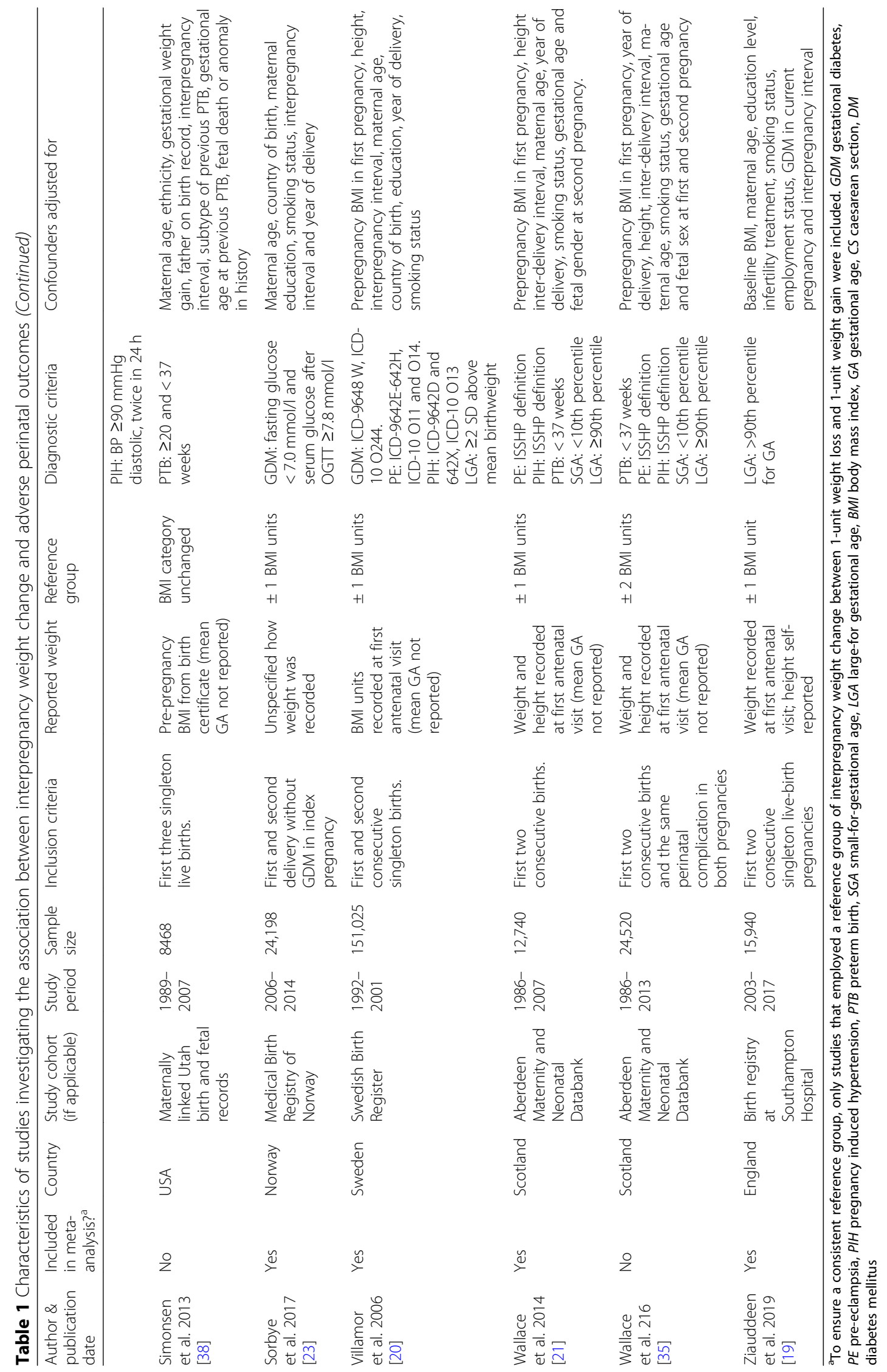


systematic reviews (www.covidence.org). The review protocol was designed a priori and registered with PROSPERO under registration number CRD42017067326.

\section{Study selection}

Studies were selected using the following predetermined inclusion criteria: [i] interpregnancy weight change reported in kilogram $(\mathrm{kg})$, BMI units $\left(\mathrm{kg} / \mathrm{m}^{2}\right)$ or percentage body weight change in multiparous women with any time interval between the consecutive births, [ii] any of the perinatal outcomes of interest in the subsequent pregnancy, and [iii] observational, cohort or case-controlled human study design with a sample size $\geq 50$, that were reported in English. When studies reported data from overlapping study populations, the study with the largest sample size was selected for inclusion. Information extracted from each study included country of research, study cohort name (if applicable), study period, sample size, study inclusion criteria, methods of weight reporting, definition of reference group, diagnostic criteria for perinatal outcomes and demographics that studies adjusted for. All study selection, full text screening, and data extraction was undertaken independently by two researchers (NEWDT and KLM), following PRISMA guidelines [7]. Disagreements were decided through a third opinion (AMW).

\section{Data synthesis}

Interpregnancy weight change was defined as the difference between pre-pregnancy weight in the index

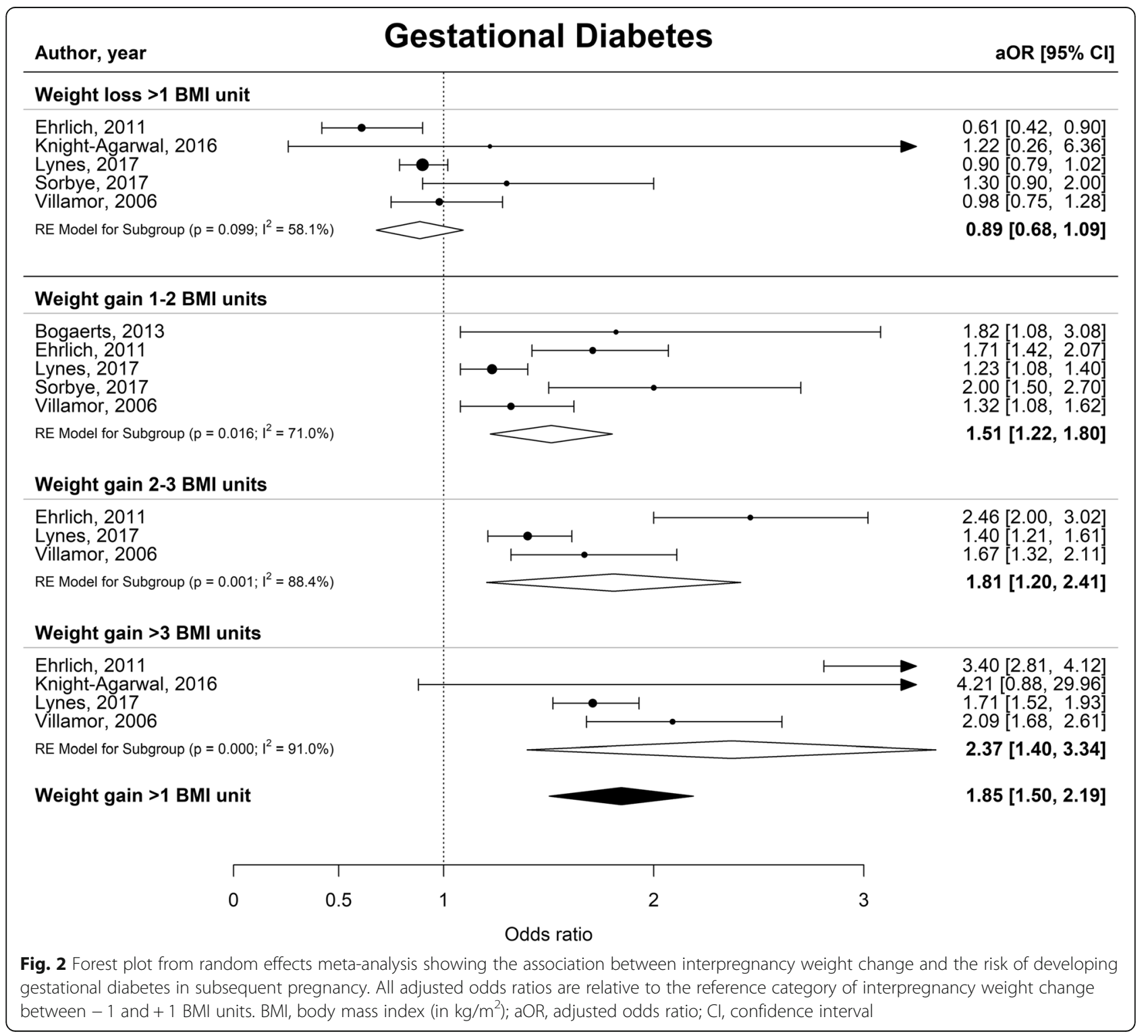


pregnancy, defined as the earliest recorded pregnancy, and pre-pregnancy weight in the subsequent pregnancy. Interpregnancy weight gain and loss were defined on two categorical scales; (i) for the meta-analysis we utilised categories of $>1$ BMI unit interpregnancy weight loss, BMI gain between 1 and 2 units, BMI gain between 2 and 3 units or BMI gain of more than 3 units and (ii) for the dose-response analysis we utilised a BMI change of $0,1,2$ or $3+$ units. Crude odds ratios (calculated from studies providing relevant counts) and adjusted odds ratios for each outcome of interest were extracted from the selected publications.

Random effects meta-analysis was used to synthesize the odds ratios for weight change categories. To ensure a consistent reference group, only studies that employed a reference group of interpregnancy weight change between 1-unit weight loss and 1-unit weight gain were included. Heterogeneity was assessed using the $\mathrm{I}^{2}$ statistic.

We conducted a separate analysis comparing interpregnancy weight change and the risk of developing adverse perinatal outcomes in women with a normal BMI $\left(<25 \mathrm{~kg} / \mathrm{m}^{2}\right)$ versus women with an overweight BMI $(\geq 25 \mathrm{~kg} / \mathrm{m} 2)$, at the start of their index pregnancy. To do so, adjusted odds ratios for both BMI categories were extracted from the publications and summarised by random effects meta-analysis.

Dose-response relationships were assessed by plotting association measurements from studies providing multiple weight gain categories. Where ranges of BMI changes were reported, the midpoint of the category was

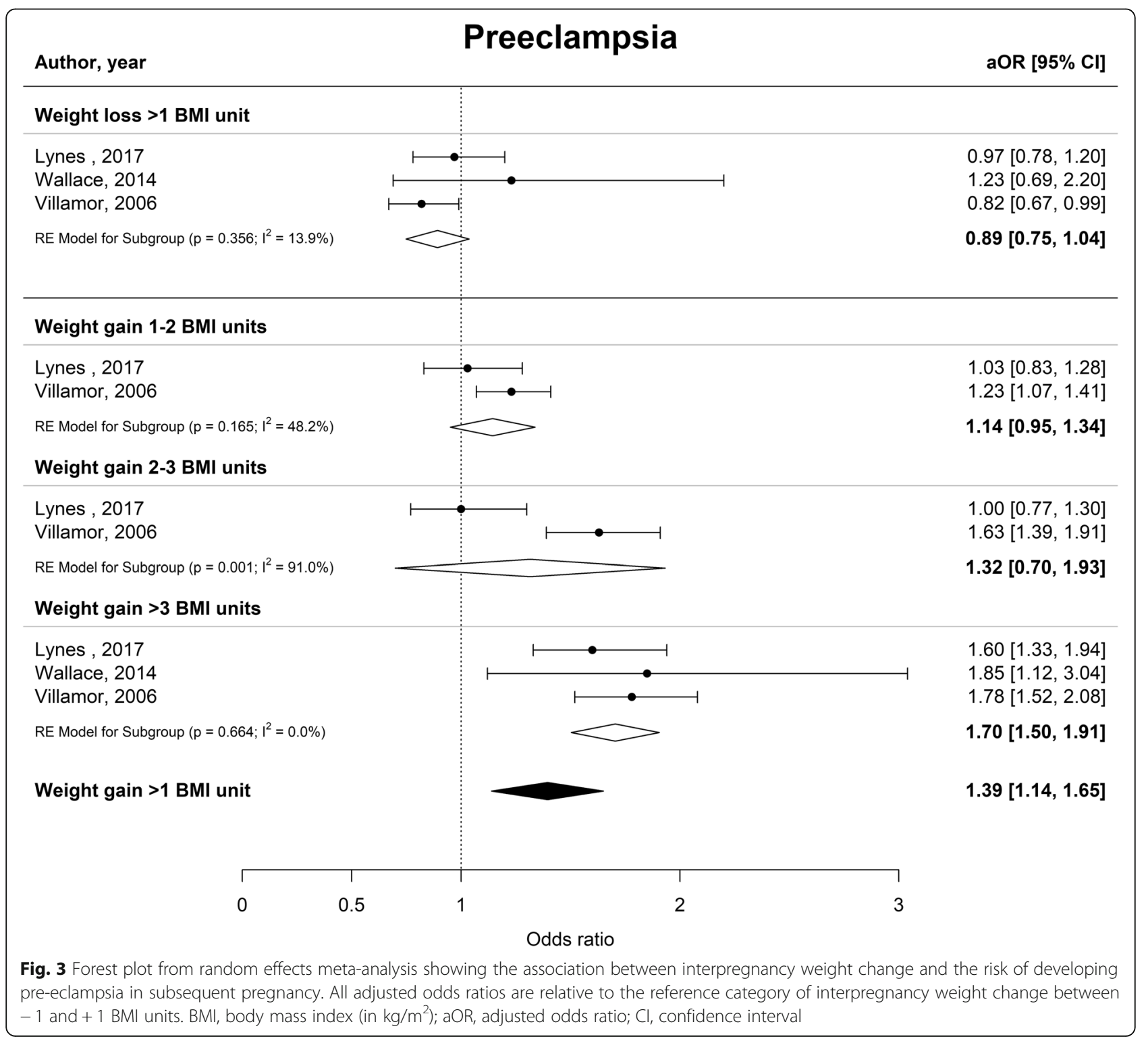


used (e.g. 1.5 BMI units change for the category weight change between 1 and 2 BMI units).

Statistical analysis and graphical presentation were performed using the metafor package in R for Windows, version 3.4.2.

\section{Assessment of risk of bias}

A sensitivity analysis was undertaken to assess potential impact of bias in individual studies by excluding studies that scored below 5 out of 9 points in the Newcastle-Ottawa Scale (NOS [8]) quality scoring assessment (Additional file 4: Table S2). Furthermore, leave-one-study-out analyses were conducted to identify whether one study leveraged the overall effect size estimate.

\section{Results}

Study selection

We identified and screened 4500 unique publications and included 194 articles for full text review (Fig. 1). A total of 27 studies were eligible for inclusion. Three studies were excluded due to overlapping study populations [9-11] and one was excluded because of a sample size $<50$ women [12]. From the remaining 23 studies selected to take forward, a total of 671,906 women were identified for inclusion in the review (Table 1). Eighteen studies included only nulliparous women at the index pregnancy. The proportion of women older than 35 years varied between studies from 3 to $33 \%$. All studies were conducted in Western populations, although this was not an inclusion criterion. Seven studies, comprising of 280,672 women, were included in the meta-analysis.

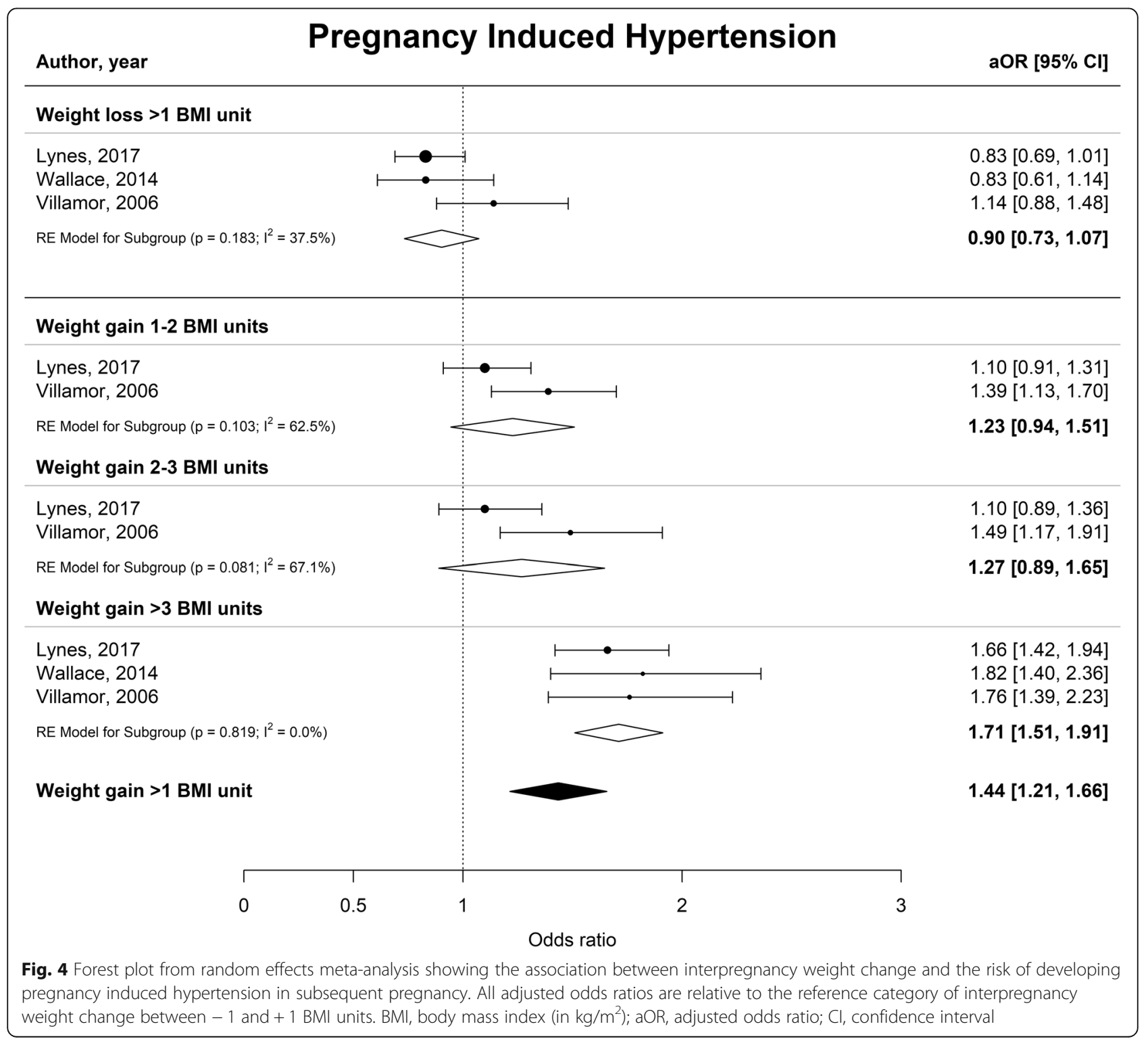




\section{Synthesis of results}

Interpregnancy weight gain of between 1 and 2 BMI units was associated with a $51 \%$ higher risk of developing GDM (aOR 1.51 [1.22-1.80], $\mathrm{I}^{2}=70.1 \%$ ), whereas an increase of 2-3 or more than $3 \mathrm{BMI}$ units was associated with an 81 and 137\% higher risk (aOR 1.81 [1.20-2.41], $\mathrm{I}^{2}=88.4 \%$ and aOR 2.37 [1.50-3.34], $\mathrm{I}^{2}=91.0 \%$ respectively) (Fig. 2). Furthermore, interpregnancy weight gain of more than 3 BMI units was associated with a higher risk of PE or PIH (aOR 1.70 [1.50-1.91], $\mathrm{I}^{2}=0.0 \%$ and aOR $1.71[1.51-1.91] \mathrm{I}^{2}=0.0 \%$ respectively) (Figs. 3 and 4 ). The association between interpregnancy weight change and the risk of delivering an LGA neonate could only be estimated for a weight gain $>3$ BMI units, and showed a 63\% higher risk (aOR 1.63 [1.30-1.97], $\mathrm{I}^{2}=$ 85.6\%) (Fig. 5). In contrast, interpregnancy weight loss of $>1$ BMI unit was associated with a lower risk of delivering an LGA neonate, (aOR 0.79 [0.58-0.99], $\mathrm{I}^{2}=$ 86.1\%) (Fig. 5), but there was no conclusive evidence of association of interpregnancy weight loss with the risk of developing GDM, PE or PIH (Figs. 2, 3 and 4). There was an insufficient number of studies to conduct a meta-analysis on adjusted odds ratios for the outcomes of SGA and PTB. A meta-analysis combining the crude odds ratios rather than adjusted ratios showed a significant higher risk of developing PE (COR 1.31 [1.091.53], $\mathrm{I}^{2}=75.1 \%$ ), but showed similar results for the association between interpregnancy weight gain and the risk of developing GDM, PE or PIH (Additional file 1: Figure S1 for interpregnancy weight loss and Additional file 2: Figure S2 for weight gain). For the outcomes of SGA and PTB, meta-analyses of crude odds

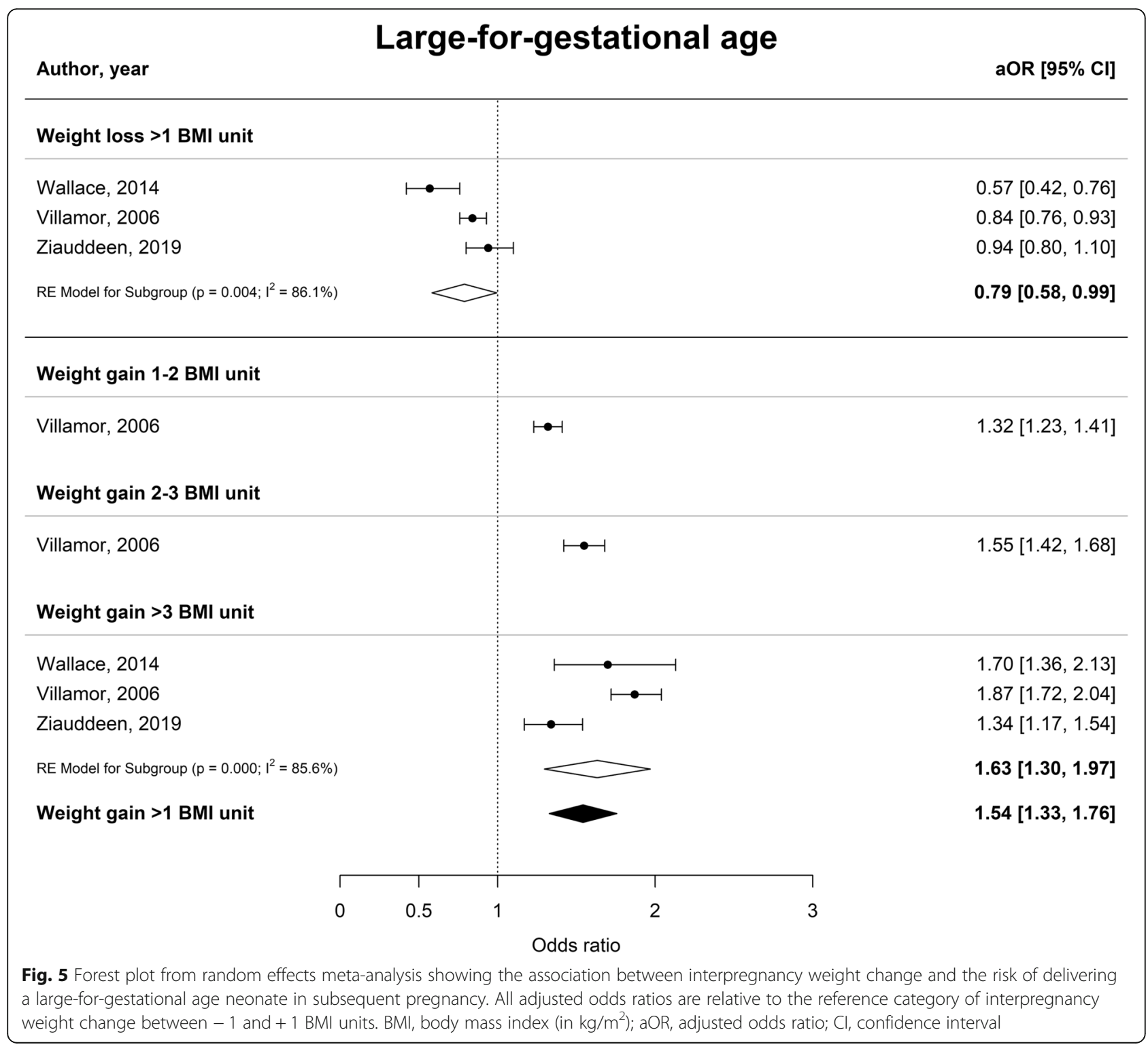




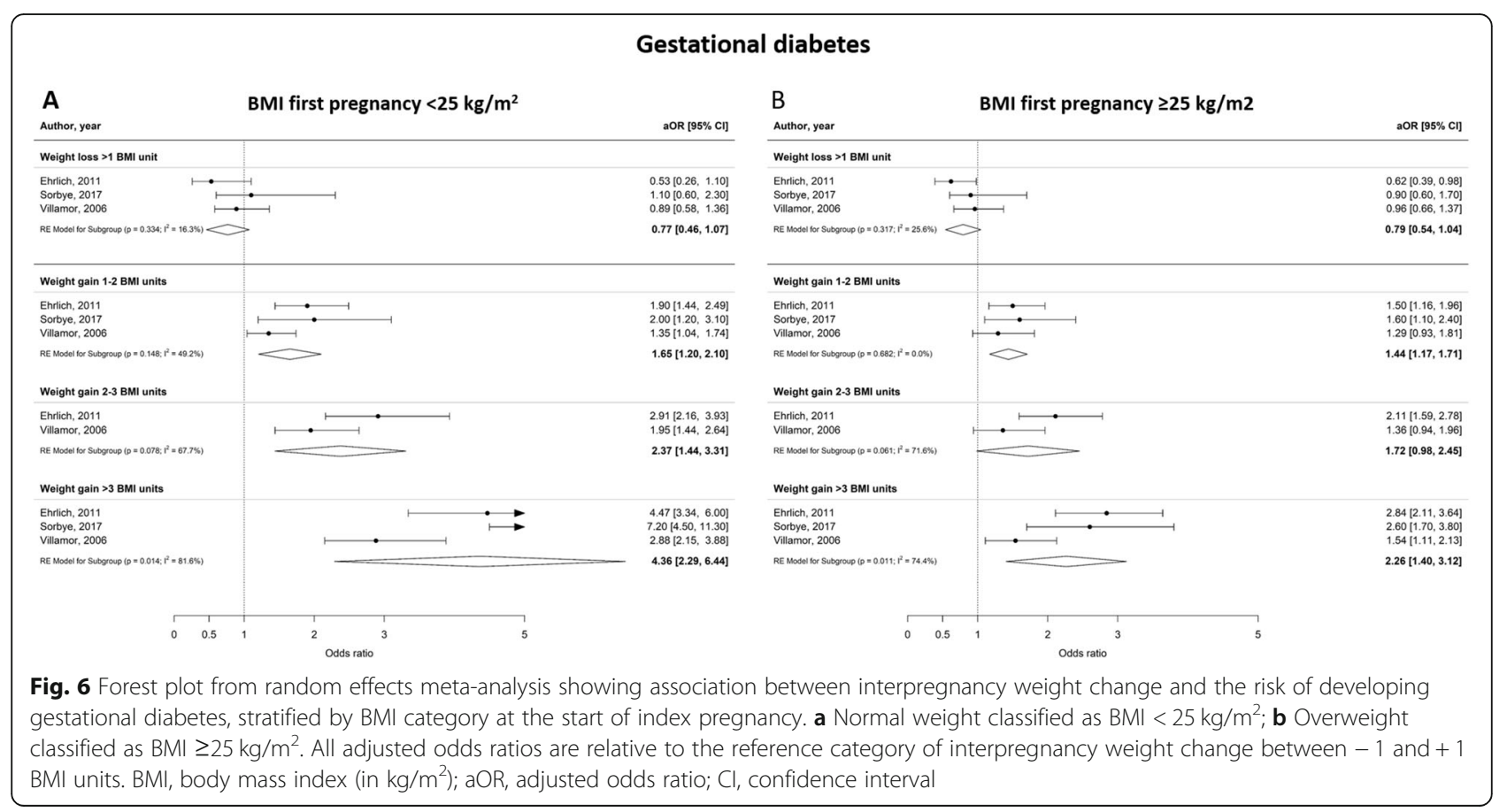

ratios showed interpregnancy weight loss of $>1$ BMI unit was associated with a higher risk of delivering a SGA neonate or delivering preterm (cOR 1.53 [1.35$1.71], \mathrm{I}^{2}=0.0 \%$ and $\left.\mathrm{cOR} 1.45[1.21-1.69], \mathrm{I}^{2}=26.7 \%\right]$ respectively), but there was no evidence of association with interpregnancy weight gain (Additional file 1: Figure S1 and Additional file 2: Figure S2).
Figures 6, 7 and 8 show the odds ratios for the risk of developing an adverse perinatal outcome after interpregnancy weight gain, stratified by BMI category in the index pregnancy (normal weight; BMI $<25 \mathrm{~kg} / \mathrm{m}^{2}$ versus overweight; BMI $\left.\geq 25 \mathrm{~kg} / \mathrm{m}^{2}\right)$. Women with a normal weight at the start of the index pregnancy had a higher risk of developing GDM after interpregnancy weight

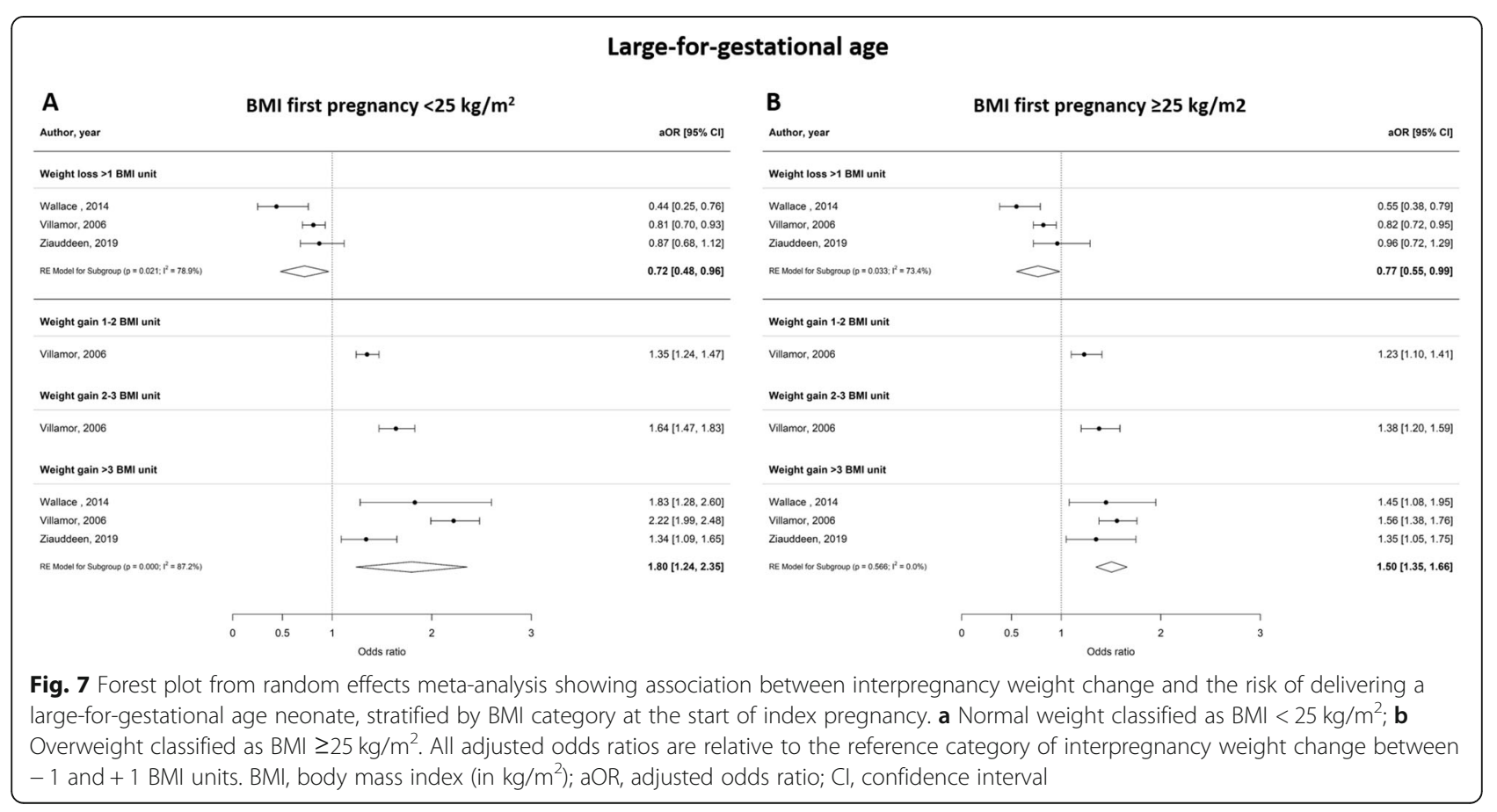




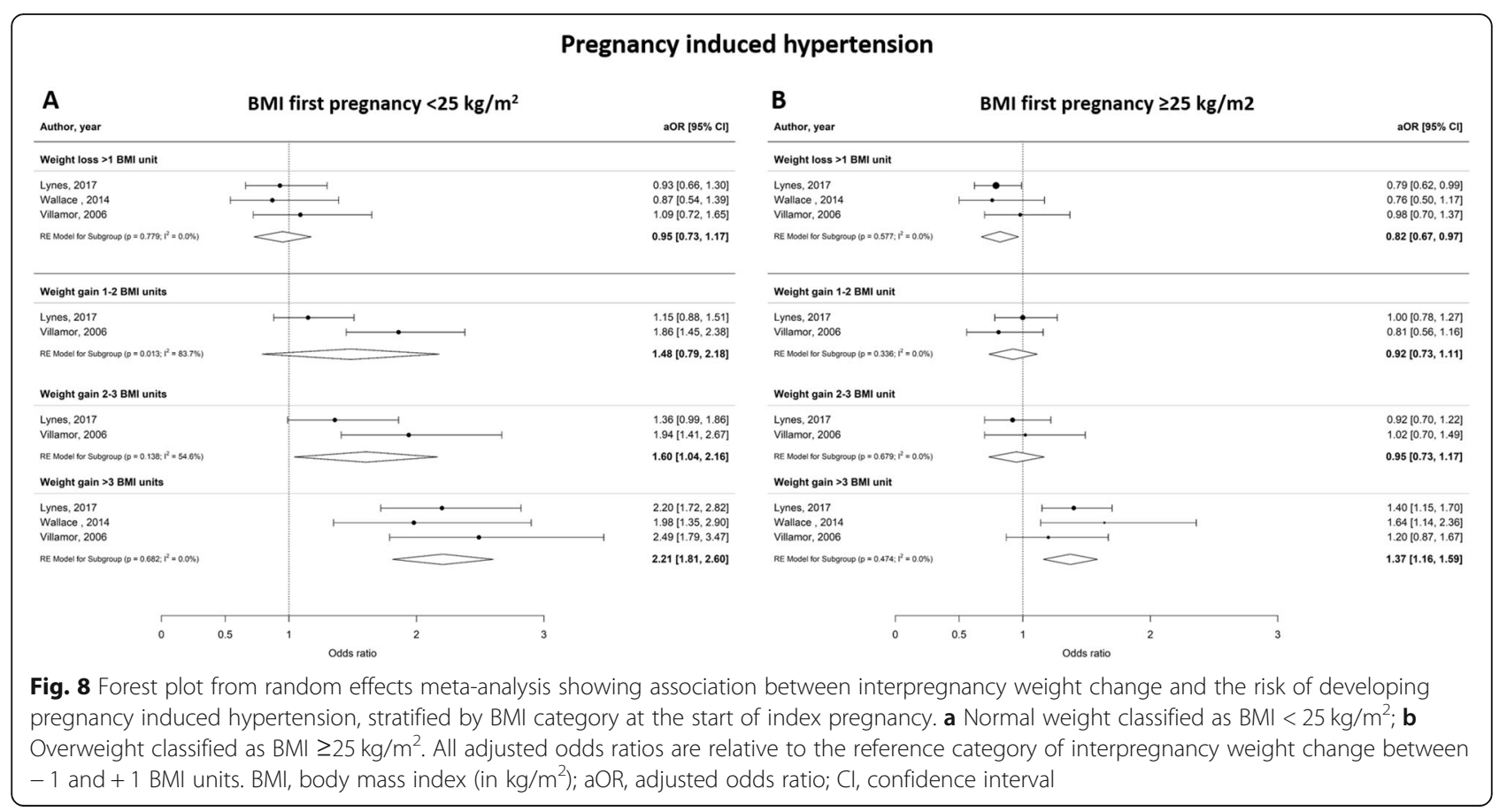

gain > 3 BMI units (aOR 4.36 [2.29-6.44], $\mathrm{I}^{2}=81.6 \%$ ) compared to women with an overweight BMI (aOR 2.26 [1.40-3.12], $\mathrm{I}^{2}=74.4 \%$ ) (Fig. $6 \mathrm{a}$ and b). Similarly, women with a BMI $<25 \mathrm{~kg} / \mathrm{m}^{2}$ were at higher risk of delivering a LGA neonate after interpregnancy weight gain $>3$ BMI units than women with BMI $\geq 25 \mathrm{~kg} / \mathrm{m}^{2}$ (aOR 1.80 [1.24-2.35], $\mathrm{I}^{2}=87.2 \%$ versus aOR 1.50 [1.35-1.66], $\mathrm{I}^{2}=$ $0.0 \%$ respectively) (Fig. $7 \mathrm{a}$ and $\mathrm{b}$ ). Women with a normal BMI at the start of their index pregnancy were at higher risk of developing PIH in a subsequent pregnancy after interpregnancy weight gain of 2-3 BMI (aOR 1.60 [1.042.16, $\mathrm{I}^{2}=54.6 \%$ ) and > 3 BMI units (aOR 2.21 [1.81-2.60], $\left.\mathrm{I}^{2}=0.0 \%\right)$, compared to women with an overweight BMI (2-3 units gain; aOR 0.95 [0.73-1.17], $\mathrm{I}^{2}=0.0 \%,>3$ units gain; aOR 1.37 [1.16-1.59], $\mathrm{I}^{2}=0.0 \%$ ) (Fig. 8a and b). We did not find differential effects of interpregnancy weight loss between women with a normal BMI and women with an overweight BMI on the risk of developing GDM, PIH or delivering an LGA neonate.

There was an approximate log-linear association between interpregnancy weight gain and the risk of developing GDM (Fig. 9a), PE (Fig. 9b) or PIH (Fig. 9c) and delivering a LGA neonate (Fig. 9d).

\section{Risk of bias of included studies}

After assessing the study selection criteria, comparability of cases and controls and outcome assessments through the NOS, we identified four studies of poor quality (NOS score $<5$, Additional file 4: Table S2). However, as these studies did not employ a reference group of \pm 1 BMI unit, they were already excluded from the meta- analyses. Leave-one-out-analyses showed that removing the study by Villamor et al. made the association between GDM and interpregnancy weight change of $2-3$ or $>3$ BMI units not significant. We did not find evidence that the outcomes for PE or PIH were driven by one study. For the outcome of delivering an LGA neonate, leave-one-out analyses could not be conducted due to only two studies being included in the meta-analysis.

\section{Discussion}

\section{Main findings}

This study systematically summarises and examines the published literature on the associations between interpregnancy weight change and several common perinatal outcomes. Our main findings are that interpregnancy weight gain is associated with a higher risk of developing GDM, PE, PIH and delivering an LGA neonate, while interpregnancy weight loss is associated with a lower risk of delivering an LGA neonate. BMI at the start of the index pregnancy possibly modifies the risk of developing GDM, PIH or delivering an LGA neonate after interpregnancy weight gain. Furthermore, we identify a positive approximately log-linear relationship between interpregnancy weight gain and the risk of developing GDM, PE, PIH or delivering a LGA neonate.

\section{Comparison with existing literature}

Our study confirms the associations between interpregnancy weight gain and the risk of developing GDM and LGA, as also shown in a recent metaanalysis [13]. Our research additionally summarises 


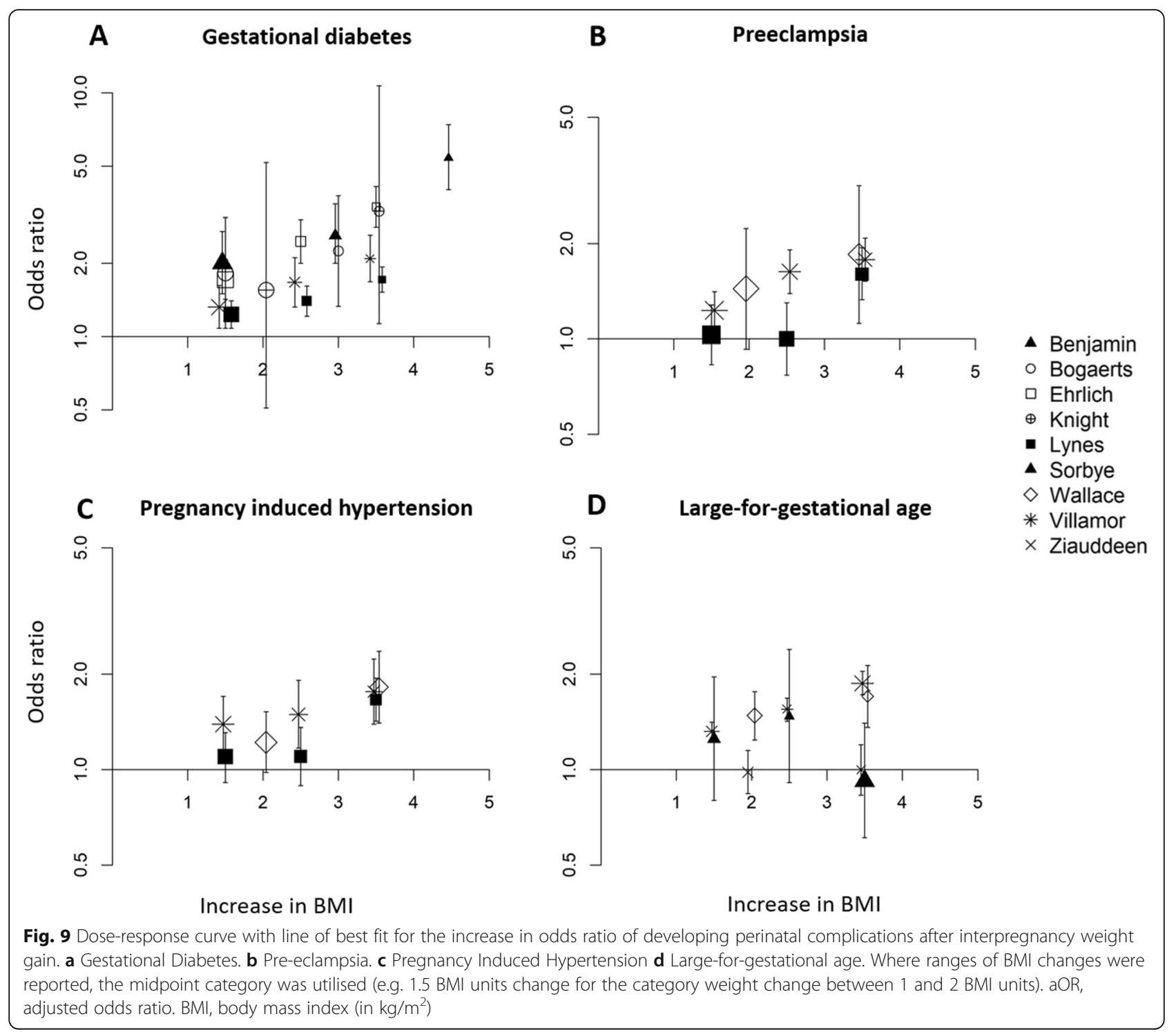

the effect of interpregnancy weight change on the risk of developing hypertensive disorders in pregnancy. However, our meta-analysis is to the authors knowledge the first study to show that gaining weight between pregnancies increases the risk of developing hypertensive disorders in the subsequent pregnancy. The observation that starting BMI possibly modifies this association is important for women with a healthy BMI at the start of their index pregnancy, as research often emphasises the risk associated with being overweight or obese, and women with a healthy BMI might not be aware of the risk that comes with (small) interpregnancy weight gain. Although the risks of (excessive) gestational weight gain [14] and high prepregnancy BMI $[15,16]$ on perinatal outcomes are well understood, the effects of interpregnancy weight gain are relatively unknown and are essential to understand in order to guide women in periconception and perinatal weight management.

Our study shows an approximate log-linear association between BMI gain and the risk of developing GDM or hypertensive disorders in pregnancy. This result contributes towards understanding the association between maternal weight and pregnancy complications. Linear dose-response associations are established between obesity and the incidence of GDM, PE and PIH [17], between adiposity and pre-eclampsia [18], as well as maternal weight and pre-eclampsia [15] and GDM [16]. Our identified associations emphasise the detrimental effects of (small amounts of) weight gain, additional to the influence of absolute BMI. This can contribute towards understanding the importance of postpartum weight management and highlights the need for the development of clinical guidelines. 


\section{Strengths and limitations}

A strength of our study is we ensured a homogenous reference group (i.e. a BMI change $\leq 1 \mathrm{~kg} / \mathrm{m}^{2}$ ) for our meta-analysis rather than including studies with very different reference groups [13]. Furthermore, we only harmonised studies reporting adjusted odds ratios, which all considered maternal age, country of origin, social economic status and smoking status as potential confounders. Nevertheless, our study has several limitations. First, between-study heterogeneity remained, arising from differences in outcome definitions and demographics, such as parity and age, and potentially differences in length of interpregnancy intervals and prevalence of perinatal complications. Of the studies selected for meta-analysis, only Lynes et al. did not restrict to nulliparous women, although removing this study had little impact on the results. Second, GDM, PE and $\mathrm{PIH}$ were either not defined in publications or the definitions of these adverse outcomes differed between studies, hence caution is needed when comparing effect estimates between studies. Third, it was not possible to consistently assess the impact of previous pregnancy complications, which may lead to excessive interpregnancy weight changes and a higher risk of subsequent pregnancy complications. Fourth, studies varied in the way they measured pre-pregnancy weight, with the majority of studies using selfreported weight (and height) to calculate BMI and interpregnancy weight change. Although evidence suggests that maternal reports of pre-pregnancy weight are in general consistent with clinical records [42], bias due to systematic over- or underreporting cannot be excluded. We can also not exclude the possibility of publication bias, as this could not be assessed due to the small number of studies available per adverse outcome and funnel plot assessment is generally not recommended with less than 10 studies [43]. Lastly, we were unable to make the distinction between spontaneous preterm birth and medically induced preterm birth. We hypothesise that an increased risk of preterm birth is at least partly related to the increased risk of carrying an SGA neonate, as (suspected) growth restriction is one of the main causes of medically induced premature birth [44]. However, inadequate nutrition in the context of severe maternal weight loss could also contribute to a higher risk of both SGA and preterm birth [45].

\section{Conclusions}

Our study highlights the importance of postpartum weight management, but also identifies opportunities for future research. There is a need to capture the typical weight change profiles of women in various BMI classes, to further elucidate risk groups. This will support further research into weight management strategies, eventually aiming to implement evidencebased weight control interventions to benefit maternal and offspring health. It is particularly important to elucidate strategies for postpartum weight loss in normal weight women, as this group might not be the focus of current research and interventions, yet may be at highest risk of adverse outcomes from interpregnancy weight gain.

In conclusion, we show that interpregnancy weight gain impacts on the risk of developing perinatal complications in a subsequent pregnancy and it is possible that BMI at the index pregnancy modifies these associations. These findings highlight the need to encourage women to return to their pre-pregnancy weight before conceiving again in an effort to reduce the risk of perinatal complications. Future work should focus on defining the most effective strategies to achieve this outcome.

\section{Supplementary information}

Supplementary information accompanies this paper at https://doi.org/10. 1186/s12884-019-2566-2.

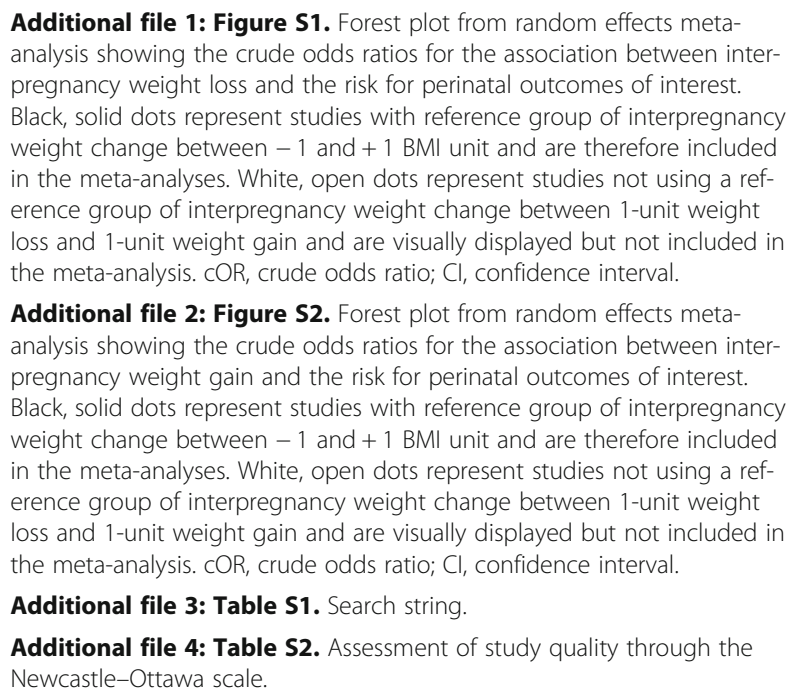
pregnancy weight loss and the risk for perinatal outcomes of interest. Black, solid dots represent studies with reference group of interpregnancy weight change between -1 and +1 BMI unit and are therefore included in the meta-analyses. White, open dots represent studies not using a reference group of interpregnancy weight change between 1-unit weight loss and 1-unit weight gain and are visually displayed but not included in the meta-analysis. COR, crude odds ratio; $\mathrm{Cl}$, confidence interval.

Additional file 2: Figure S2. Forest plot from random effects metaanalysis showing the crude odds ratios for the association between interpregnancy weight gain and the risk for perinatal outcomes of interest. Black, solid dots represent studies with reference group of interpregnancy weight change between -1 and +1 BMI unit and are therefore included in the meta-analyses. White, open dots represent studies not using a reference group of interpregnancy weight change between 1-unit weight loss and 1-unit weight gain and are visually displayed but not included in the meta-analysis. cOR, crude odds ratio; $\mathrm{Cl}$, confidence interval.

Additional file 3: Table S1. Search string.

Additional file 4: Table S2. Assessment of study quality through the Newcastle-Ottawa scale.

\section{Abbreviations \\ BMI: Body mass index; Cl: Confidence interval; CS: Caesarean section; GDM: Gestational diabetes mellitus; PE: Preeclampsia; PIH: Pregnancy induced hypertension; NOS: Newcastle-Ottawa scale; aOR: adjusted odds ratio; COR: crude odds ratio; PRISMA: Preferred reporting items for systematic reviews and meta-analysis PTBPreterm birth; LGA: Large-for-gestational age; SGA: Small-for-gestational age}

\section{Acknowledgements}

This work was supported by core funding from: the UK Medical Research Council (MR/L003120/1), the British Heart Foundation (RG/13/13/30194; RG/ 18/13/33946) and the National Institute for Health Research [Cambridge Biomedical Research Centre at the Cambridge University Hospitals NHS Foundation Trust] [*].

*The views expressed are those of the authors and not necessarily those of the NHS, the NIHR or the Department of Health and Social Care. 


\section{Authors' contributions}

NEWDT, CEA, SEO and AMW made substantial contributions to the conception of the project. NEWDT and KLM developed the protocol, AMW advised on the protocol design. NEWDT and KLM managed the systematic search and screened papers for inclusion with consultation from AMW. NEWDT and KLM extracted the data and assessed the risk of bias in included studies. NEWDT preformed the statistical analysis and wrote the first draft of the manuscript. All authors contributed to interpretation of the data, substantively revised the manuscript and approved the final version.

\section{Funding}

NEWDT is sponsored by a British Heart Foundation PhD Scholarship under the reference FS/16/53/32729. KLM is funded by the NIHR Blood and Transplant Research Unit in Donor Health and Genomics (NIHR BTRU-201410024). SEO is supported by the British Heart Foundation (RG/17/12/33167) and Medical Research Council (MC_UU_12012/4). CEA is supported by an Isaac Newton Trust/Wellcome Trust ISSF/ University of Cambridge Joint Research Grant. The funding bodies did not play any role in the design of the study, collection, analysis and interpretation of the data, nor in the writing of the manuscript.

\section{Availability of data and materials}

All data generated or analysed during this study are included in this published article and its additional file.

\section{Ethics approval and consent to participate}

Not applicable

\section{Consent for publication}

Not applicable

\section{Competing interests}

The authors declare that they have no competing interests.

\section{Author details}

'Department of Public Health and Primary Care, University of Cambridge, 2 Worth's Causeway Cambridge, Cambridge CB1 8RN, UK. ${ }^{2}$ University of Cambridge Metabolic Research Laboratories and MRC Metabolic Diseases Unit, Wellcome Trust-MRC Institute of Metabolic Science, Addenbrooke's Hospital, Cambridge CB2 OQQ, UK. ${ }^{3}$ Department of Obstetrics and Gynaecology, University of Cambridge, Box 223, The Rosie Hospital and NIHR Cambridge Comprehensive Biomedical Research Centre, Cambridge, UK.

\section{Received: 6 August 2019 Accepted: 22 October 2019}

Published online: 28 October 2019

\section{References}

1. Organisation WH. Fact sheet: Obesity and overweight reviewed February 2018 [Available from: http://www.who.int/mediacentre/factsheets/fs311/en/. Accessed 22 July 2019

2. Vahratian A. Prevalence of overweight and obesity among women of childbearing age: results from the 2002 National Survey of family growth. Matern Child Health J. 2009;13(2):268-73.

3. Marchi J, Berg M, Dencker A, Olander EK, Begley C. Risks associated with obesity in pregnancy, for the mother and baby: a systematic review of reviews. Obes Rev. 2015;16(8):621-38.

4. Han Z, Mulla S, Beyene J, Liao G, McDonald SD, Knowledge SG. Materna underweight and the risk of preterm birth and low birth weight: a systematic review and meta-analyses. Int J Epidemiol. 2011;40(1):65-101.

5. Excellence. NIfHaC. Weight management before, during and after pregnancy. 2010

6. Whitworth $M$, Dowswell T. Routine pre-pregnancy health promotion for improving pregnancy outcomes. Cochrane Database Syst Rev. 2009;7(4): CD007536.

7. Moher D, Liberati A, Tetzlaff J, Altman DG, Group P. Preferred reporting items for systematic reviews and meta-analyses: the PRISMA statement. Ann Intern Med. 2009:151(4):264-9 W64.

8. Stang A. Critical evaluation of the Newcastle-Ottawa scale for the assessment of the quality of nonrandomized studies in meta-analyses. Eur J Epidemiol. 2010;25(9):603-5.
9. Bhattacharya S, Campbell DM, Smith NC. Pre-eclampsia in the second pregnancy: does previous outcome matter? Eur J Obstet Gynecol Reprod Biol. 2009;144(2):130-4

10. Mostello D, Jen Chang J, Allen J, Luehr L, Shyken J, Leet T. Recurrent preeclampsia: the effect of weight change between pregnancies. Obstet Gynecol. 2010;116(3):667-72.

11. Wallace JM, Bhattacharya S, Horgan GW. Weight change across the start of three consecutive pregnancies and the risk of maternal morbidity and SGA birth at the second and third pregnancy. PLoS One. 2017;12(6):e0179589.

12. Lu GC, Luchesse A, Chapman V, Cliver S, Rouse DJ. Screening for gestational diabetes mellitus in the subsequent pregnancy: is it worthwhile? Am J Obstet Gynecol. 2002;187(4):918-21.

13. Oteng-Ntim E, Mononen S, Sawicki O, Seed PT, Bick D, Poston L. Interpregnancy weight change and adverse pregnancy outcomes: a systematic review and meta-analysis. BMJ Open. 2018;8(6):e018778.

14. Goldstein RF, Abell SK, Ranasinha S, Misso M, Boyle JA, Black MH, et al. Association of Gestational Weight Gain with Maternal and Infant Outcomes: a systematic review and meta-analysis. JAMA. 2017;317(21):2207-25.

15. O'Brien TE, Ray JG, Chan WS. Maternal body mass index and the risk of preeclampsia: a systematic overview. Epidemiology. 2003;14(3):368-74.

16. Torloni MR, Betran AP, Horta BL, Nakamura MU, Atallah AN, Moron AF, et al. Prepregnancy BMI and the risk of gestational diabetes: a systematic review of the literature with meta-analysis. Obes Rev. 2009;10(2):194-203.

17. Trojner Bregar A, Tul N, Fabjan Vodusek V, Verdenik I, Lucovnik M, Jansa $V$, et al. A dose-response relation exists between different classes of pre-gravid obesity and selected perinatal outcomes. Arch Gynecol Obstet. 2017;296(3):465-8.

18. Wang Z, Wang P, Liu H, He X, Zhang J, Yan H, et al. Maternal adiposity as an independent risk factor for pre-eclampsia: a meta-analysis of prospective cohort studies. Obes Rev. 2013;14(6):508-21.

19. Ziauddeen N, Wilding S, Roderick PJ, Macklon NS, Alwan NA. Is maternal weight gain between pregnancies associated with risk of large-forgestational age birth? Analysis of a UK population-based cohort. BMJ Open. 2019:9(7):e026220.

20. Villamor E, Cnattingius S. Interpregnancy weight change and risk of adverse pregnancy outcomes: a population-based study. Lancet. 2006;368(9542): 1164-70.

21. Wallace JM, Bhattacharya S, Campbell DM, Horgan GW. Inter-pregnancy weight change impacts placental weight and is associated with the risk of adverse pregnancy outcomes in the second pregnancy. BMC Pregnancy Childbirth. 2014;14:40

22. Lynes C, McLain AC, Yeung EH, Albert P, Liu J, Boghossian NS. Interpregnancy weight change and adverse maternal outcomes: a retrospective cohort study. Ann Epidemiol. 2017;27(10):632-7 e5.

23. Sorbye LM, Skjaerven R, Klungsoyr K, Morken NH. Gestational diabetes mellitus and interpregnancy weight change: a population-based cohort study. PLoS Med. 2017;14(8):e1002367.

24. Ehrlich SF, Hedderson MM, Feng J, Davenport ER, Gunderson EP, Ferrara A Change in body mass index between pregnancies and the risk of gestational diabetes in a second pregnancy. Obstet Gynecol. 2011;117(6): 1323-30.

25. Bogaerts A, Van den Bergh BR, Ameye L, Witters I, Martens E, Timmerman D, et al. Interpregnancy weight change and risk for adverse perinatal outcome. Obstet Gynecol. 2013;122(5):999-1009.

26. Chen A, Klebanoff MA, Basso O. Pre-pregnancy body mass index change between pregnancies and preterm birth in the following pregnancy. Paediatr Perinat Epidemiol. 2009;23(3):207-15

27. Cheng CJ, Bommarito K, Noguchi A, Holcomb W, Leet T. Body mass index change between pregnancies and small for gestational age births. Obstet Gynecol. 2004;104(2):286-92.

28. Getahun D, Ananth CV, Oyelese Y, Chavez MR, Kirby RS, Smulian JC. Primary preeclampsia in the second pregnancy: effects of changes in prepregnancy body mass index between pregnancies. Obstet Gynecol. 2007:110(6):1319-25.

29. Getahun D, Ananth CV, Peltier MR, Salihu HM, Scorza WE. Changes in prepregnancy body mass index between the first and second pregnancies and risk of large-for-gestational-age birth. Am J Obstet Gynecol. 2007;196(6): 530 e1-8.

30. Hoff GL, Cai J, Okah FA, Dew PC. Pre-pregnancy overweight status between successive pregnancies and pregnancy outcomes. J Women's Health (Larchmt). 2009;18(9):1413-7. 
31. Jain AP, Gavard JA, Rice JJ, Catanzaro RB, Artal R, Hopkins SA. The impact of interpregnancy weight change on birthweight in obese women. Am J Obstet Gynecol. 2013;208(3):205 e1-7.

32. Knight-Agarwal $C R$, Williams LT, Davis $D$, Davey $R$, Cochrane $T$, Zhang $H$, et al. Association of BMl and interpregnancy BMl change with birth outcomes in an Australian obstetric population: a retrospective cohort study. BMJ Open. 2016;6(5):e010667.

33. Kruse AR, Darling MS, Hansen MK, Markman MJ, Lauszus FF, Wielandt HB. Recurrence of gestational diabetes in primiparous women. Acta Obstet Gynecol Scand. 2015;94(12):1367-72.

34. McBain RD, Dekker GA, Clifton VL, Mol BW, Grzeskowiak LE. Impact of interpregnancy BMI change on perinatal outcomes: a retrospective cohort study. Eur J Obstet Gynecol Reprod Biol. 2016;205:98-104.

35. Wallace JM, Bhattacharya S, Campbell DM, Horgan GW. Inter-pregnancy weight change and the risk of recurrent pregnancy complications. PLoS One. 2016;11(5):e0154812.

36. Glazer NL, Hendrickson AF, Schellenbaum GD, Mueller BA. Weight change and the risk of gestational diabetes in obese women. Epidemiology. 2004; 15(6):733-7.

37. Pole JD, Dodds LA. Maternal outcomes associated with weight change between pregnancies. Can J Public Health. 1999;90(4):233-6.

38. Simonsen SE, Lyon JL, Stanford JB, Porucznik CA, Esplin MS, Varner MW. Risk factors for recurrent preterm birth in multiparous Utah women: a historical cohort study. BJOG. 2013;120(7):863-72.

39. Benjamin RH, Littlejohn S, Canfield MA, Ethen MK, Hua F, Mitchell LE. Interpregnancy change in body mass index and infant outcomes in Texas: a population-based study. BMC Pregnancy Childbirth. 2019;19(1):119.

40. Bastian LA, West NA, Corcoran C, Munger RG. Cache County study on memory $\mathrm{H}$, aging. Number of children and the risk of obesity in older women. Prev Med. 2005;40(1):99-104.

41. Rooney BL, Schauberger CW. Excess pregnancy weight gain and long-term obesity: one decade later. Obstet Gynecol. 2002;100(2):245-52.

42. Lederman SA, Paxton A. Maternal reporting of prepregnancy weight and birth outcome: consistency and completeness compared with the clinical record. Matern Child Health J. 1998:2(2):123-6.

43. Lau J, loannidis JP, Terrin N, Schmid CH, Olkin I. The case of the misleading funnel plot. Bmj. 2006:333(7568):597-600.

44. Goldenberg RL, Culhane JF, lams JD, Romero R. Epidemiology and causes of preterm birth. Lancet. 2008:371(9606):75-84.

45. Neggers Y, Goldenberg RL. Some thoughts on body mass index, micronutrient intakes and pregnancy outcome. J Nutr. 2003;133(5 Suppl 2): 1737S-40S.

46. Bender W, Hirshberg A, Levine LD. Interpregnancy Body Mass Index Changes: Distribution and Impact on Adverse Pregnancy Outcomes in the Subsequent Pregnancy. Am J Perinatol. 2019;36(05):517-21.

47. Crosby DA, Walsh JM, Segurado R, McAuliffe FM. Interpregnancy weight changes and impact on pregnancy outcome in a cohort of women with a macrosomic first delivery: a prospective longitudinal study. BMJ Open. 2017: 7(5):e016193.

\section{Publisher's Note}

Springer Nature remains neutral with regard to jurisdictional claims in published maps and institutional affiliations.

Ready to submit your research? Choose BMC and benefit from:

- fast, convenient online submission

- thorough peer review by experienced researchers in your field

- rapid publication on acceptance

- support for research data, including large and complex data types

- gold Open Access which fosters wider collaboration and increased citations

- maximum visibility for your research: over $100 \mathrm{M}$ website views per year

At $\mathrm{BMC}$, research is always in progress.

Learn more biomedcentral.com/submissions 